\title{
Divergent Temporal Response of Abundant and Rare Bacterial Communities to Transient Escherichia coli 0157:H7 Invasion
}

OPEN ACCESS

Edited by:

Aymé Spor,

INRA UMR 1347 Agroécologie,

France

Reviewed by:

Jincai Ma,

Jilin Unversity, China

Shuo Jiao,

Northwest A\&F University, China

*Correspondence:

Zhiyuan Yao

yaozhiyuan@nbu.edu.cn

tThese authors have contributed equally to this work and share first authorship

Specialty section: This article was submitted to

Terrestrial Microbiology, a section of the journal Frontiers in Microbiology

Received: 08 February 2021 Accepted: 22 April 2021 Published: 07 June 2021

Citation: Zhang N, Liang C, Liu X, Yao Z, Zhu DZ, Du S and Zhang H (2021)

Divergent Temporal Response of Abundant and Rare Bacterial Communities to Transient Escherichia coli 0157:H7 Invasion.

Front. Microbiol. 12:665380. doi: 10.3389/fmicb.2021.665380

\section{Nan Zhang ${ }^{1,2+}$, Chunling Liang ${ }^{1+}$, Xiangjun Liu', Zhiyuan Yao ${ }^{1,3 *}$, David Z. Zhu ${ }^{1,2}$, Shicong Du ${ }^{4}$ and Huajun Zhang ${ }^{4}$}

\footnotetext{
${ }^{1}$ School of Civil and Environmental Engineering, Ningbo University, Ningbo, China, ${ }^{2}$ Institute of Ocean Engineering, Ningbo University, Ningbo, China, ${ }^{3}$ Zhejiang Provincial Key Laboratory of Agricultural Resources and Environment, Hangzhou, China, ${ }^{4}$ School of Marine Sciences, Ningbo University, Ningbo, China
}

The release of Escherichia coli (E. coli) O157:H7 has been widely found in various environments, but little is known about the probable influence of the transient $E$. coli O157:H7 invasion on the native microbial community. Here, we investigated the temporal response of two bacterial biospheres (abundant and rare) of two marsh sediments against E. coli 0157:H7 during a 60-day incubation. The diversity of both biospheres showed no evident response to 0157:H7 invasion. Temporal factor exhibited greater effects on bacterial variation than $\mathrm{O} 157: \mathrm{H} 7$ invasion. We found that $\mathrm{O} 157: \mathrm{H} 7$ invasion led to an increase in the niche breadth of the bacterial community while decreasing the efficiency of bacterial interaction of the abundant taxa. Moreover, the rare biosphere exhibited enhanced stability against $\mathrm{O} 157: \mathrm{H} 7$ invasion compared with the abundant biosphere, acting as the backbone in resisting external disturbance. Furthermore, each subcommunity assembly showed different randomness levels. The stochastic events were relatively more important in constraining the abundant taxa assembly after invasion. Collectively, E. coli O157:H7 exhibited diverse tangible impact on both biospheres, which unearthed differential responses of abundant and rare biosphere against transient microbial invasion.

Keywords: Escherichia coli 0157:H7, invasion, microbial assembly, niche, rare biosphere

\section{INTRODUCTION}

The rapid spread of microorganisms into various ecological systems is known to impact the function and structural make-up of the native microbial communities (Li et al., 2019; Thakur et al., 2019). Thus, current researches in the fields of microbial ecology and invasion biology have been focused on the identification of novel microbial invasion along with the assessment of their microecological impact (Wei et al., 2018; Li et al., 2019; Braga et al., 2020).

Extensive studies have been conducted on invasion by phytopathogens since they gradually displace the resident communities, resulting in significant economic losses in agriculture (Levine et al., 2004; Robin et al., 2011; Wei et al., 2015, 2018). Furthermore, zoonotic pathogens are also 
known to invade the natural microbial communities (Van Elsas et al., 2011; Liang et al., 2019). The release of livestock feces into the soil might result in potential invasion by approximately $10^{9}$ Escherichia coli (E. coli) cells/g feces. Different from the phytopathogenic invaders, zoonotic pathogens are highly abundant in the initial phase. However, they seldom transform into autochthonous species (Ishii et al., 2006; Mallon et al., 2018). This observation raises the question whether the transient invaders, such as zoonotic pathogens, could induce a change in the resident micro-community. Recent studies showed that transient invaders altered the composition and niche structure in synthetically assembled communities of microbes (Mallon et al., 2018; Li et al., 2019; Xing et al., 2020). Since synthetic communities are simplified than their natural counterparts, more knowledge is required regarding the alteration in natural microbial community.

Bacterial communities are known to be comprised of ubiquitously abundant species as well as several rare species (Pedros-Alio, 2012). The abundant species account for a significant proportion of bacterial biomass and biogeochemical cycling, while the rare species are critical for the ecosystem stability and functions (Pedros-Alio, 2012; Jia et al., 2018; Du et al., 2020). Recent investigations have revealed how abundant and rare bacterial subcommunities respond distinctively to external disturbances, such as diatom blooms (Zhang et al., 2020), inorganic carbon stress (Shu et al., 2018), organic pollution (Jiao et al., 2017), etc. However, there is limited information on the impact of microbial invaders on rare biosphere of sediment. Thus, it is imperative to explore the response of both abundant and rare species against microbial invaders for an improved understanding of the microecological role of transient invaders in native microbial communities.

Interspecies interactions have an indispensable position in structuring the development of bacterial communities (Faust and Raes, 2012; Gao et al., 2019). The overall influence of unsuccessful invaders on the co-occurrence pattern and central species remains unclear. Additionally, the ecological processes underlying the patterns observed in relation to species abundance across time and space are vital in microbial ecology (Nemergut et al., 2013; Dini-Andreote et al., 2015). Both the deterministic and stochastic processes concurrently act for the regulation of the microbial community assembly (Nemergut et al., 2013; Zhou and Ning, 2017). However, the degree to which differences in the balance between these two processes is driven by the microbial invader needs to be investigated. Previous studies were mostly focused on a single time point after microbial invasion and did not explore the variation in microbial population with time (Mallon et al., 2018; Li et al., 2019; Xing et al., 2020). Thus, a set of successional systems post-microbial invasion would be ideal to unravel the effect of invaders on the ecological processes governing the microbial assembly.

The release of E. coli O157:H7, a common cause of foodborne illness (Van Elsas et al., 2011), into natural environment facilitates its interaction with the indigenous microorganisms (Yao et al., 2014, 2019b; Mallon et al., 2018). Our research group has previously found that $E$. coli $\mathrm{O} 157: \mathrm{H} 7$ persists in marsh sediment for 36 to 53 days (Liang et al., 2019). However, only little relevant details are available regarding the influence of intruding E. coli O157:H7 on the marsh bacterial community, which harbors high biodiversity and important ecosystem functions (Yao et al., 2019a). Here, we examined the effects of invading E. coli O157:H7 on both abundant and rare biosphere of two distinct marsh sediments by investigating the variation in diversity, composition, co-occurrence pattern, and assembly processes with time. The alterations in these parameters would correspond to identical mechanisms unraveling how different communities respond to invasion.

\section{MATERIALS AND METHODS}

\section{Sediment Description}

The surface marsh $(5-20 \mathrm{~cm})$ sediments (B and P) which was covered with distinct vegetation (bare and Phragmites australis, respectively) were collected in the estuarine marsh $\left(30^{\circ} 21^{\prime} 56^{\prime \prime} \mathrm{N}, 121^{\circ} 12^{\prime} 39^{\prime \prime} \mathrm{E}\right)$ in south Hangzhou Bay, China (Supplementary Figure 1; Yao et al., 2019a). The B sediment contained $3.4 \pm 0.4 \mathrm{~g} / \mathrm{kg}$ organic carbon, $68.5 \pm 8.8 \mathrm{~g} / \mathrm{kg}$ ammonia nitrogen, $0.2 \pm 0 \mathrm{~g} / \mathrm{kg}$ total nitrogen and had a $\mathrm{pH}$ of $6.7 \pm 0.2$. The $\mathrm{P}$ sediment contained $6.8 \pm 0.6 \mathrm{~g} / \mathrm{kg}$ organic carbon, $134.2 \pm 9.8 \mathrm{~g} / \mathrm{kg}$ ammonia nitrogen, $0.6 \pm 0.1 \mathrm{~g} / \mathrm{kg}$ total nitrogen and had a $\mathrm{pH}$ of $8.0 \pm 0.1$. No indigenous $E$. coli O157:H7 was detected in both sediments.

\section{Experimental Setup and Procedures}

Escherichia coli O157:H7 (non-pathogenic E. coli O157:H7 strain 3704 Tn5 luxCDAEB) was introduced into the sediment at $10^{7} \mathrm{CFU} / \mathrm{g}$, and the water content was adjusted to $100 \%$ waterholding capacity. This inoculation density represented a classical invading E. coli in nature, which was in agreement with previous studies (Fukushima and Seki, 2004; Yao et al., 2013, 2019b; Liang et al., 2019), providing a reliable template to examine the effects of E. coli O157 intrusion. Sediment samples inoculated with equivalent amount of sterile water served as non-invading controls. The inoculated samples were incubated in a dark environment at constant temperature $\left(25 \pm 1^{\circ} \mathrm{C}\right)$ and constantly maintained by adding sterile deionized water every 2 days to make up for the moisture loss during incubation. After 36 and 48 days, there was no culturable E. coli O157:H7 detected in B and $\mathrm{P}$ sediment (Liang et al., 2019). To discover the existence of lag time in indigenous bacterial communities in response to the invasion, we sampled 12 days after no culturable E. coli O157:H7 was detected in each sediment. Correspondingly, the sediment B was destructively sampled on days $0,5,20,36$, and 48. The sediment $P$ was destructively sampled on days $0,5,20$, 36,48 , and 60 . Each sampling, we stored approximately $10 \mathrm{~g}$ of sediment from individual treatment at a temperature of $-80^{\circ} \mathrm{C}$ for DNA extraction.

\section{DNA Extraction, PCR, and Sequencing}

The DNA isolation kit PowerSoil (MOBIO, United States) was employed for sediment DNA extraction according to the manufacturer guidelines. A NanoDrop spectrophotometer (NanoDrop Technologies Inc., United States) established the 
DNA concentration and quality. The V4 region of the bacterial 16S rRNA gene was amplified using the following primers: 515F (5'-GTGYCAGCMGCCGCGGTAA-3') and 806R (5'-GGA CTACNVGGGTWTCTAAT-3') (Parada et al., 2016). The PCR reaction was carried out in a reaction mixture of $20 \mu \mathrm{l}$ under the following thermo-cycling operations: $4^{\circ} \mathrm{C}$ for $3 \mathrm{~min}, 30$ cycles of $95^{\circ} \mathrm{C}$ for $30 \mathrm{~s}, 50^{\circ} \mathrm{C}$ for $30 \mathrm{~s}$, and $72^{\circ} \mathrm{C}$ for $45 \mathrm{~s}$. This was followed by extension at $72^{\circ} \mathrm{C}$ for $10 \mathrm{~min}$. We pooled equimolar amounts of the purified products of PCR of individual sample and sequenced them using the Illumina MiSeq platform (Illumina, Inc., San Diego, CA, United States) having a pairedend ( $2 \mathrm{bp} \times 300 \mathrm{bp})$ approach. The NCBI accession number of the raw sequencing data was PRJNA669260.

\section{Sequence Data Processing}

Raw sequencing data were processed using QIIME $2^{1}$, followed by denoising and filtration using plug-in DADA2 database. Subsequently, the Amplicon Sequence Variants (ASVs) was identified by a naïve Bayes classifier that was trained with the plug-in feature-classifier employing the 16S rRNA gene database having a $99 \%$ similarity of the SILVA 132 . Post-quality trimming, $1,398,000$ reads of high-quality remained in the dataset, yielding 16,595 ASVs. The random rarefication was carried out at the similar sequence depth (at 23,300 sequences in a single sample) to make correction for varying sampling efforts. We used this ASV table for all further analyses. Abundant and rare ASVs were categorized based on relative abundances $>0.01 \%$ per sample and $<0.005 \%$ across all samples, respectively. R v3.5.3 $3^{2}$ was used to conduct both alpha $(\alpha)$ and beta $(\beta)$ diversity analyses. We estimated the Shannon index and made use of the Weighted UniFrac distance for $\beta$-diversity. The Bray-Curtis dissimilarity of $\beta$-diversity was used for time-lag regression. Niche breadth was calculated using "spaa" package in R v3.5.3.

\section{Co-occurrence Network and Community Assembly Analysis}

To improve statistical confidence, ASVs present in at least 20\% of samples were retained for the construction of networks. Subsequently, all possible pairwise Spearman's rank correlations ( $\rho)$ between those ASVs were calculated. Only robust $(\rho>0.6$ or $\rho<-0.6)$ and statistically significant $(p<0.01)$ correlations were incorporated into network analyses. Module analysis and topology results were made with Gephi interactive platform. Modules were identified by the Louvain algorithm. Then, the topology of Erdös-Réyni random networks was calculated with same node and edge. Finally, the network visualization of dominant taxa was plotted by chord diagram.

\section{Ecological Stochasticity Estimation and Determinism in Community Assembly Analysis}

To understand bacterial community assembly processes, we calculated the estimated normalized stochasticity ratio (NST)

${ }^{1}$ https://qiime2.org/

${ }^{2} \mathrm{http}: / /$ www.r-project.org by adopting the null model to ascertain the tochasticity value in community assembly to assess the relative influence of ecological activities. If NST $<50 \%$, it represented a more deterministic assembly while NST $>50 \%$ denoted a more stochastic assembly. The "tNST" function was used to conduct this analysis with the following parameters: "dist method" of "bray," "abundance weighted" of "TRUE" and "rand" of " 1,000 " in the "NST" package.

\section{Statistical Analysis}

Significant difference in the $\alpha$-diversity and niche breadth were tested by making use of ANOVA (one-way analysis of variance) and significant difference of bacterial composition was tested by Mann-Whitney U test. All test analysis was made by SPSS statistical software (SPSS, United States). We used the Bray-Curtis dissimilarity to conduct permutational multivariate analysis of variance by using the ADONIS function to calculate the differences in community compositions among samples. Additionally, the Weighted UniFrac distances were used to perform a principal coordinates analysis ( $\mathrm{PCoA}$ ) to pinpoint the similarity among various bacterial groups. $\mathrm{R}$ statistical software was employed for the analysis unless indicated otherwise.

\section{RESULTS}

\section{Generalized Patterns of $\alpha$-Diversity and Bacterial Composition}

We classified 2,428 ASVs (14.63\%), encompassing 58.31\% of all sequences, as abundant taxa and 11,459 ASVs (69.05\%), encompassing $22.75 \%$ of all sequences, as rare taxa. The rare subcommunity showed higher level of $\alpha$-diversity compared with the abundant $(p<0.05)$, indicating a more diverse taxonomy in the rare biosphere (Supplementary Figure 2). Additionally, we found no considerable difference between the invaded group (INV) and the non-invaded control group (CON) for both sediments (Supplementary Table 1). Some phyla, including Chloroflexi and Proteobacteria, were either found profusely in every ecosystem, whereas some phyla, such as Latescibacteria, Bacteroidetes, Nitrospira, and Planctomycetes were always rare (Supplementary Figure 3 ). In addition, the abundant and rare taxa in $\mathrm{B}$ and $\mathrm{P}$ sediments show no significant difference after invasion at the phylum level (Supplementary Figure 4). We hypothesized that the invading O157:H7 did not have a direct impact on the bacterial composition regarding dominant taxa distribution and might be reflected in a lower classification level. The Venn diagram revealed considerable overlap between the INV and CON groups for the majority of abundant ASVs (Figures 1A,B), whereas the rare taxa included most of the treatment-specific ASVs (Figures 1C,D). For the abundant taxa in the $\mathrm{B}$ and $\mathrm{P}$ sediments, more ASVs belonging to Verrucomicrobia and Chloroflexi were found in the INV group (Figures 1A,B). Additionally, the dominant role of $\gamma$-proteobacteria in the CON group was replaced by other phylum in B sediment (Figure 1A). However, we did not observe a consistent change between the $\mathrm{B}$ and $\mathrm{P}$ sediments in the rare taxa (Figures 1C,D). 

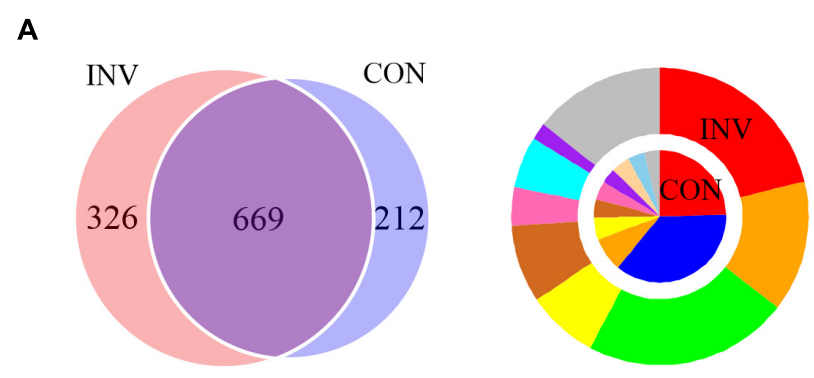

C
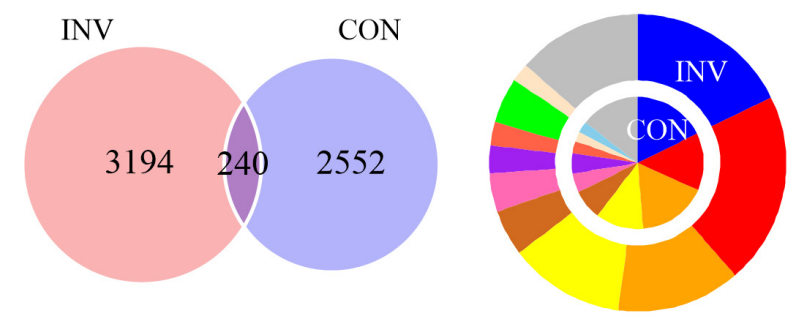

Acidobacteria

Chloroflexi

Gammaproteobacteria

Patescibacteria
Actinobacteria

Cyanobacteria

Gemmatimonadetes

Planctomycetes
B
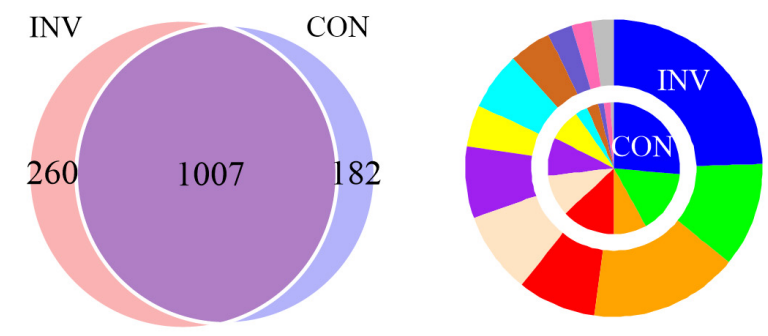

D
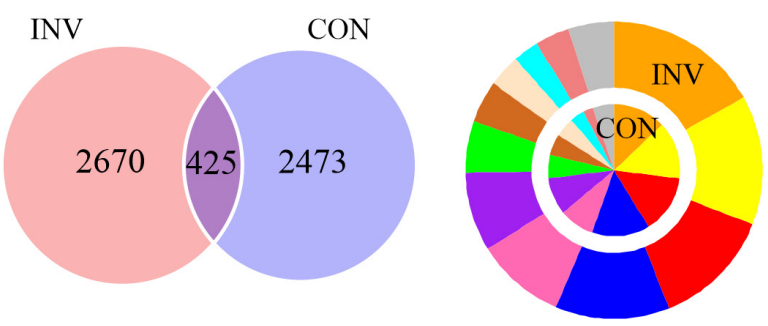

Alphaproteobacteria

Bacteroidetes

Deltaproteobacteria

Firmicutes

Latescibacteria

Verrucomicrobia
Nanoarchaeaeota

Others

FIGURE 1 | Venn diagram representing the numbers of shared and amplicon sequence of variants (ASVs) among the CON and the INV group. The average relative abundances of the phyla (relative abundance above $2 \%$ ) in each special category are shown in the pie chart. CON, the control group; INV, the invasion group. (A) Abundant taxa of the B sediment (B) Abundant taxa of the P sediment (C) Rare taxa of the B sediment (D) Rare taxa of the P sediment.

\section{Successions of Abundant and Rare Taxa After 0157:H7 Invasion}

Principal coordinates analysis (PCoA) based on Weighted Unifrac distance was carried out to analyze the successional trajectories of whole bacterial structure (Figure 2). All samples were primarily clustered by time (ADONIS, $\mathrm{R}^{2}=0.023$, $p=0.013$ ) rather than by invasion treatment (ADONIS, $\left.R^{2}=0.016, p=0.641\right)$, indicating that the temporal factor primarily regulated bacterial succession. Relatively apparent but not significant division between the INV and CON group was found in both abundant and rare sub communities in $\mathrm{B}$ and $\mathrm{P}$ sediments in the late stage (Supplementary Figure 5). The temporal turnover of abundant and rare subcommunities after O157:H7 invasion was further evaluated by the bacterial timedecay relationship (Figure 3 ). The temporal turnover of the INV group showed significant slopes and its rate was higher than the CON group, except the rare taxa of the $\mathrm{P}$ invasion group (Figure 3), suggesting that the bacterial communities of the INV group were undergoing a directional change. Also, $\beta$-diversity was attributed to richness of species instead of their replacement (turnover) based on the partition of $\beta$-diversity, which facilitated the transition in community constitution (Supplementary Figure 6). Finally, we estimated the niche breadths of community-level habitat to further explore the patterns of $\beta$-diversity (Figure 4). All samples had a greater value of niche breadth for the abundant taxa in comparison with the rare taxa (Figure 4). Moreover, the niche breadth values of the INV group were significantly higher than the corresponding CON group $(p<0.05)$ (Figure 4).

\section{Ecological Network Analysis on the Bacterial Community}

Network analyses were done to examine the impact of microbial invasion on bacterial co-occurrence (Figures 5, 6 and Supplementary Figure 7). The observed modularity and average clustering coefficient were all greater than those of their respective Erdös-Réyni random networks and smallworld coefficient $>1$, indicating that the network had "smallworld" properties and modular structure (Table 1). The major topological characteristics of the networks were estimated to reveal the differences in co-occurrence pattern caused by O157:H7 invasion (Table 1). The high modularity values $(>0.4)$ of all the networks implied the presence of modular architecture in all the samples (Barberán et al., 2012). Thus, the microbial invasion resulted in higher modularity for both subcommunities (Table 1). Additionally, both abundant and rare taxa showed 

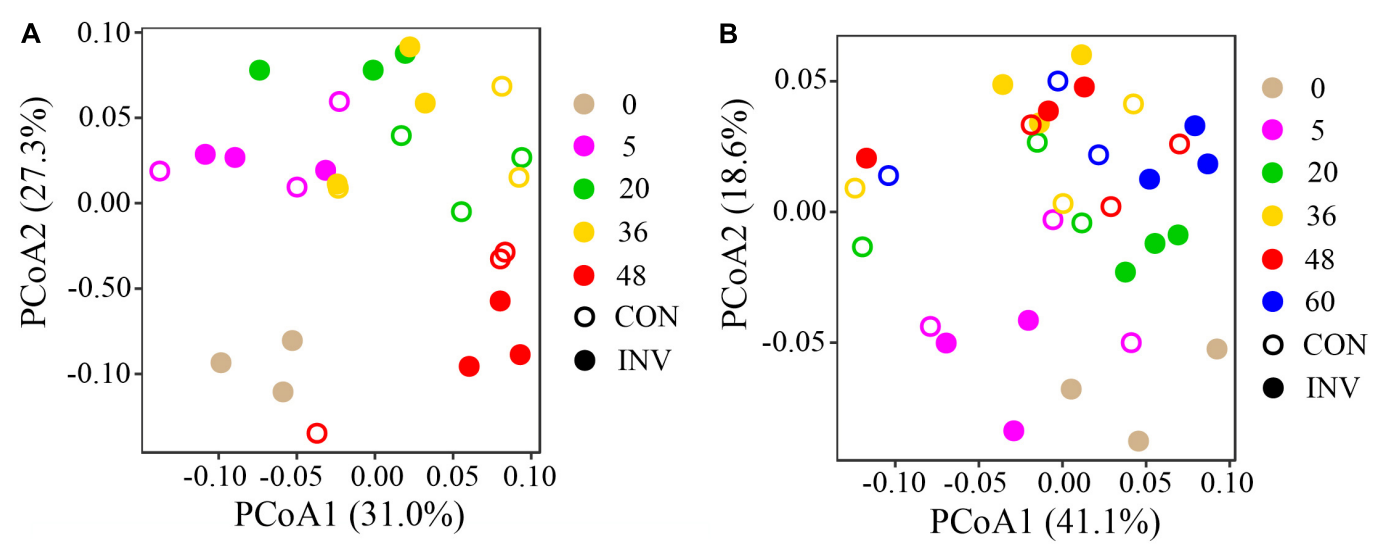

FIGURE 2 | Principal coordinate analysis (PCoA) of the bacterial communities based on the Weighted Unifrac distance. (A) B sediment (B) P sediment. CON, the control group; INV, the invasion group.
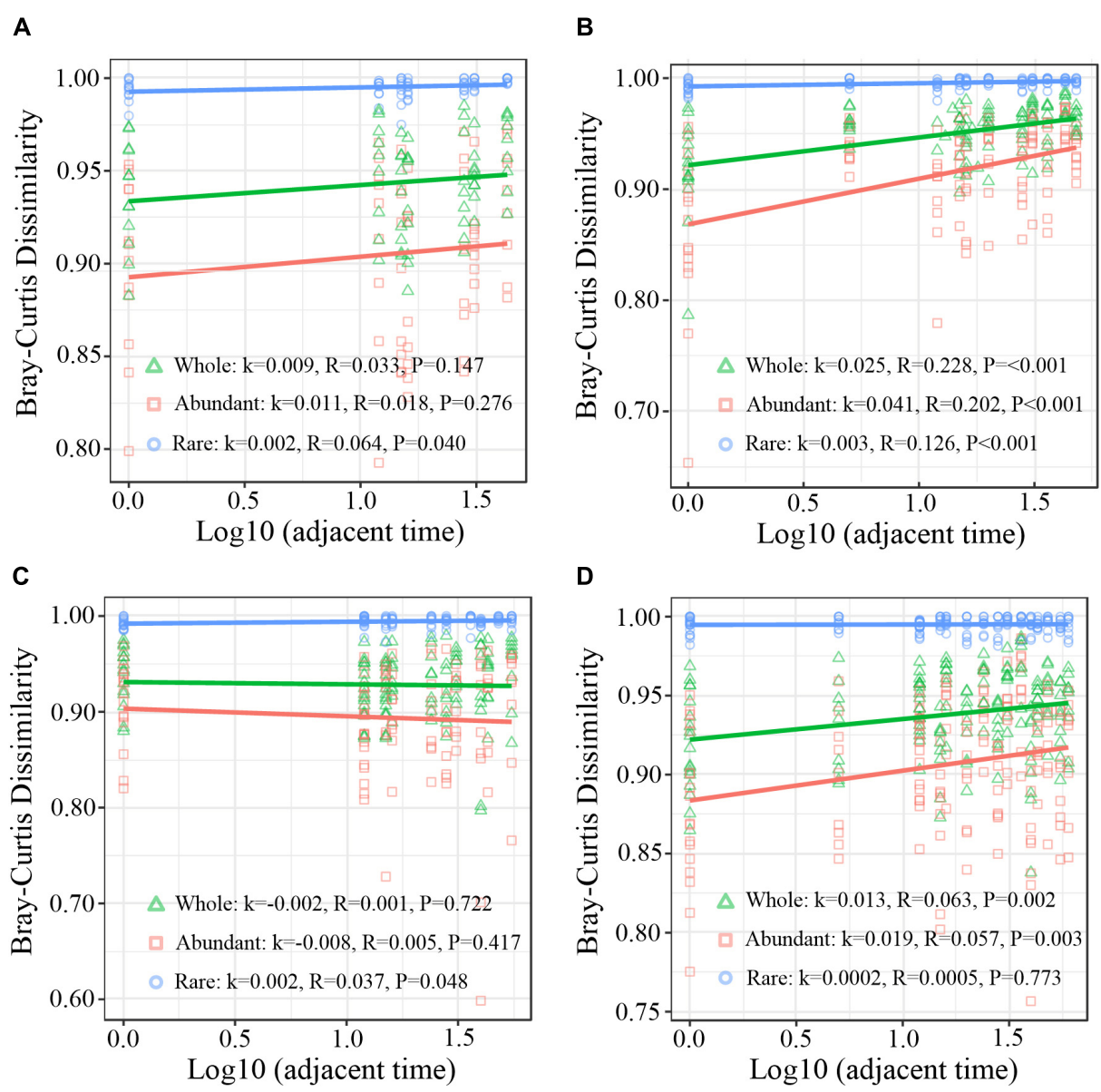

FIGURE 3 | Time-lag regression analysis of the relationship among sampling time and Bray-Curtis dissimilarity. (A) B sediment of the CON group (B) B sediment of the INV group (C) P sediment of the CON group (D) P sediment of the INV group. $k$, the regression slope; Whole, all bacterial community; Rare, rare taxa; Abundant, abundant taxa; CON, the control group; INV, the invasion group.

slightly higher network densities in the CON group than in the INV group, indicating the presence of a more complex network in the CON group. The average clustering coefficient was lower in the abundant taxa of the INV group than the CON group, while no difference was found in the rare taxa (Table 1). Moreover, the average path length, which exhibited an opposite 

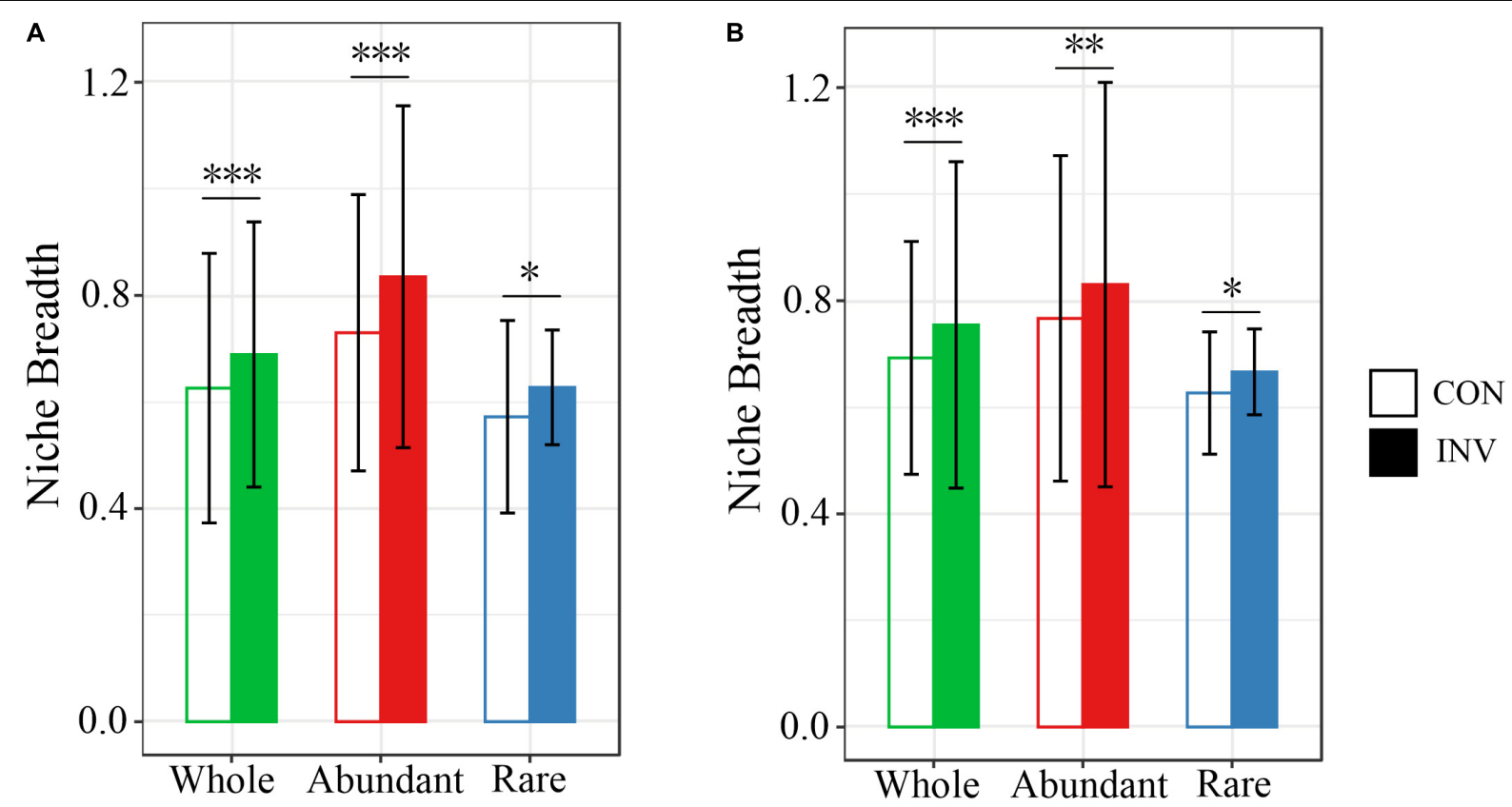

FIGURE 4 | Barplots showing the mean niche breadth for the whole, abundant, and rare bacterial communities of the B (A) and P (B) sediments. The significance level after evaluation through the nonparametric Mann-Whitney $U$ test $\left({ }^{*} p<0.05 ;{ }^{* *} p=0.001 ;{ }^{* * *} p<0.001\right)$.

trend, was much higher in the INV group than the CON group, except the rare taxa.

\section{Ecological Assembly of Bacterial Communities}

The neutral community model (NCM) estimates that a small ratio of the link between the frequency occurrence of ASVs and their relative abundance variations, ranging from 18.0-32.0\% (Supplementary Figure 8). For any treatment, we consider a frequent occurrence of several bacterial taxa (below or above the neutral prediction) than predicted by the NCM based on their gross abundance in the metacommunity (Supplementary Figures 8, 9). Next, the null model-based NST was calculated based on the phylogenetic and taxonomic metrics to further investigate the community assembly mechanism shaping the bacterial community (Figure 7). Almost the estimated NST for both abundant and rare taxa $>50 \%$. Additionally, the NST of rare taxa remained constant after microbial invasion, while that for the abundant taxa decreased.

\section{DISCUSSION}

The pollutants impact the soil bacterial community through diverse mechanisms, and the microorganisms often respond rapidly to these external changes (Jiao et al., 2016, 2017; Guo et al., 2020). E. coli O157:H7 invasion has been known to modify the structure of native indigenous community, resulting in enhanced microbial diversity as well as niche breadth (Yao et al., 2014; Mallon et al., 2018). Here, we had hypothesized that E. coli O157:H7 would alter the community diversity. However, we found no evidence regarding any significant influence of this microbial invader on the overall community diversity, structure, and composition of the marsh sediment bacterial communities (Figure 2, Supplementary Table 1, and Supplementary Figures 2, 4, 5). This observation contradicted the results of previous studies that involved synthetic communities (Mallon et al., 2018; Xing et al., 2020), which might be attributed to the increased biodiversity of native microbial communities compared with the synthetic communities (Xing et al., 2020). Also, it was possible that there were experimental effects which were not detected in this study. For example, perhaps the temporal scale of sampling (before invasion and then 5 days after the invasion) was too coarse and a more immediate sampling was needed to detect community response. We suspected that if communities were altered in the short term by E. coli O157:H7, the effects would be transient. In addition, there might be changes in the frequency of certain traits without an accompanying change in community membership. 16S rRNA genes sequencing would not detect such an effect, which require further metagenomic or functional analyses. Variation in bacterial $\beta$-diversity across samples can be ascribed to the sampling time. This indicated that postmicrobial invasion, time was a considerably important factor in regulating the bacterial succession in the microcosms. However, the bacterial structure showed more obvious dispersion during the late stage in both sediments (Figure 2). We hypothesized that the impact of the microbial invader was not visible immediately and might have lasting effects on the bacterial community. This was consistent with Amor et al. (2020) who found that transient invaders could induce long-term shifts in complex microbial communities. 
A

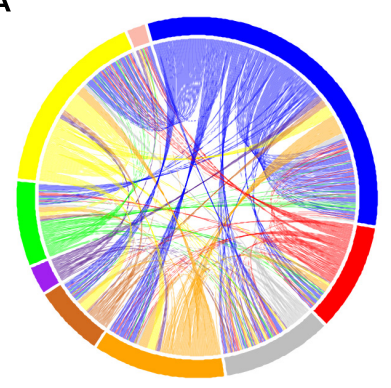

C

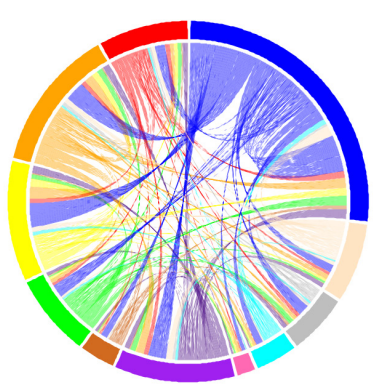

B

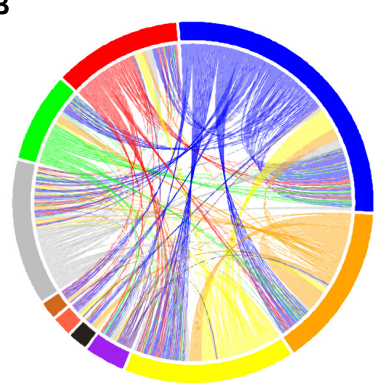

D

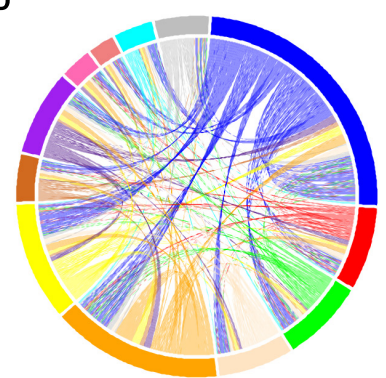

Deltaproteobacteria Gammaproteobacteria

Bacteroidetes

Acidobacteria

Latescibacteria

Chloroflexi

Planctomycetes

Thaumarchaeota

Firmicutes
Actinobacteria

Epsilonbacteraeota

Alphaproteobacteria

Verrucomicrobia

Gemmatimonadetes

Others

FIGURE $\mathbf{5}$ | Co-occurrence patterns of abundant bacterial community. The profiles of co-occurrence links among dominant taxa. Connections are colored based on the most dominant taxon (relative abundance above 2\%). (A) B sediment of the CON group (B) B sediment of the INV group (C) P sediment of the CON group (D) P sediment of the INV group.

A

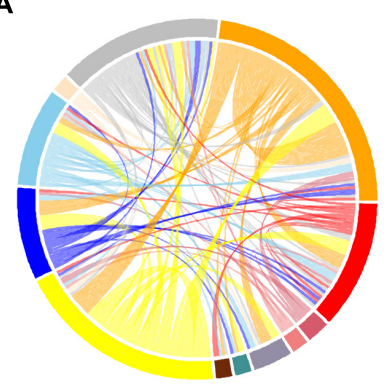

C

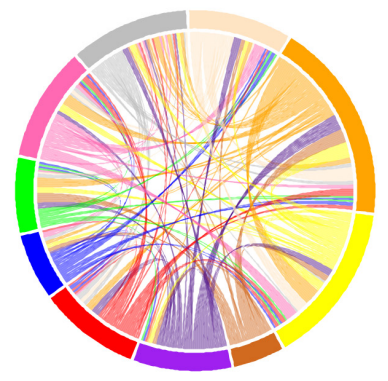

B

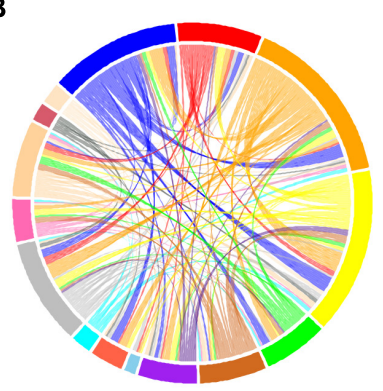

D

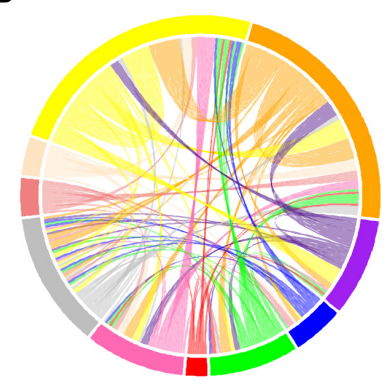

\begin{tabular}{l|l}
\hline Deltaproteobacteria & Gammaproteobacteria \\
\hline Bacteroidetes & Actinobacteria \\
\hline Acidobacteria & Patescibacteria \\
\hline Latescibacteria & Alphaproteobacteria \\
\hline Chloroflexi & Verrucomicrobia \\
\hline Planctomycetes & Gemmatimonadetes \\
\hline Nanoarchaeaeota & Others \\
\hline Firmicutes & Nitrospirae \\
Spirochaetes & Crenarchaeota \\
Elusimicrobia & \\
\hline
\end{tabular}

FIGURE 6 | Co-occurrence patterns of rare bacterial community. The profiles of co-occurrence links among dominant taxa. Connections are colored based on the most dominant taxon (relative abundance above 2\%). (A) B sediment of the CON group (B) B sediment of the INV group (C) P sediment of the CON group (D) P sediment of the INV group.

Our results showed the presence of common abundant taxa within the INV and the CON groups (Figures 1A,B), while a different distribution was observed for the rare taxa (Figures 1C,D). These results agreed with previous studies that abundant taxa were always distributed among various habitats (Du et al., 2020; Zhang et al., 2020). In addition, abundant taxa occupied broader niches than the rare ones (Figure 4), availing an array of resources supporting their survival. Furthermore, 
TABLE 1 | Topological properties of the real co-occurrence networks of whole, abundant, and rare communities and their associated random networks.

\begin{tabular}{|c|c|c|c|c|c|c|c|c|c|c|c|c|}
\hline \multirow[b]{2}{*}{ Group } & & \multicolumn{7}{|c|}{ Real networks } & \multicolumn{4}{|c|}{ Random networks } \\
\hline & & Nodes $^{a}$ & Edges $^{b}$ & Modularityc & $\begin{array}{c}\text { Average } \\
\text { clustering } \\
\text { coefficient }^{d}\end{array}$ & $\begin{array}{c}\text { Network } \\
\text { diameter }^{e}\end{array}$ & $\begin{array}{l}\text { Average } \\
\text { path } \\
\text { length }^{f}\end{array}$ & $\begin{array}{c}\text { Network } \\
\text { densities }^{g}\end{array}$ & Modularity & $\begin{array}{c}\text { Average } \\
\text { clustering } \\
\text { coefficient }\end{array}$ & $\begin{array}{c}\text { Average } \\
\text { path } \\
\text { length }\end{array}$ & $\begin{array}{l}\text { Small-world } \\
\text { coefficient }^{h}\end{array}$ \\
\hline \multirow[t]{4}{*}{ Whole } & B-CON & 577 & 2,253 & 0.953 & 0.978 & 4.00 & 1.02 & 0.014 & 0.284 & 0.013 & 3.32 & 246 \\
\hline & B-INV & 818 & 3,418 & 0.953 & 0.862 & 16.0 & 4.86 & 0.010 & 0.259 & 0.011 & 3.40 & 54.8 \\
\hline & P-CON & 1,031 & 4,979 & 0.921 & 0.823 & 38.0 & 13.4 & 0.009 & 0.233 & 0.010 & 3.31 & 20.4 \\
\hline & P-INV & 1,196 & 4,582 & 0.967 & 0.875 & 60.0 & 18.2 & 0.006 & 0.267 & 0.006 & 3.71 & 29.7 \\
\hline \multirow[t]{4}{*}{ Abundant } & B-CON & 312 & 585 & 0.946 & 0.969 & 3.00 & 1.02 & 0.012 & 0.447 & 0.011 & 4.39 & 378 \\
\hline & B-INV & 373 & 622 & 0.968 & 0.959 & 6.00 & 1.10 & 0.009 & 0.489 & 0.007 & 4.88 & 610 \\
\hline & P-CON & 504 & 1,041 & 0.937 & 0.959 & 8.00 & 1.43 & 0.008 & 0.428 & 0.007 & 4.52 & 432 \\
\hline & P-INV & 685 & 1,404 & 0.957 & 0.784 & 17.0 & 4.99 & 0.006 & 0.443 & 0.005 & 4.81 & 151 \\
\hline \multirow[t]{4}{*}{ Rare } & $\mathrm{B}-\mathrm{CON}$ & 96.0 & 169 & 0.897 & 1.00 & 1.00 & 1.00 & 0.037 & 0.403 & 0.019 & 3.51 & 185 \\
\hline & B-INV & 145 & 252 & 0.923 & 1.00 & 2.00 & 1.00 & 0.024 & 0.481 & 0.003 & 3.92 & 1,292 \\
\hline & P-CON & 163 & 260 & 0.925 & 1.00 & 1.00 & 1.00 & 0.020 & 0.493 & 0.004 & 4.38 & 1,096 \\
\hline & P-INV & 163 & 212 & 0.951 & 1.00 & 1.00 & 1.00 & 0.016 & 0.587 & 0.006 & 5.29 & 882 \\
\hline
\end{tabular}

a Number of ASVs with the correlation $r>0.6$ or $r<-0.6$ and statistical significance $(P$-value $<0.01)$.

${ }^{b}$ Number of strong and significant correlations between nodes.

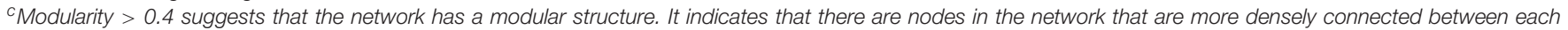
other than with the rest of the network and that their density is noticeably higher than the graph's average.

${ }^{d}$ How nodes are embedded in their neighborhood, and the degree to which nodes tend to cluster together.

e The maximum distance between all possible pairs of nodes.

${ }^{f}$ The average number of steps along the shortest paths for all possible pairs of network nodes.

$g$ The density of a graph is the ratio of the number of edges and the number of possible edges.

' Small-word coefficient > 1 indicates "small-world" properties, that is, high interconnectivity and high efficiency.

we observed a decrease in the $\gamma$-proteobacteria (class of E. coli O157:H7) in the abundant taxa of INV group (Figures 1A,B), due to niche competition because of the similar resource preferences with O157:H7, and this might trigger that the taxa better utilize or look out for niches that have not been exploited by O157:H7 (Mallon et al., 2018; Wu et al., 2021). Similar results have been communicated for Pseudomonas invasion in soil system, which reduced the relative abundance of resident bacteria which exploited the same resources (Fliessbach et al., 2009; Gómez et al., 2016). On the contrary, more abundant taxa affiliated to Chloroflexi and Verrucomicrobia, which are involved in the carbon cycle (Bergmann et al., 2011; Hug et al., 2013) were observed in the INV group (Figures 1A,B). Similarly, Wu et al. (2021) revealed that $E$. coli invasion resulted in enhanced relative abundance of Chloroflexi in residual biofilms of emergent aquatic plants. Furthermore, the change in community compositions post-invasion resulted in shifts in the niche structure as well as an increased niche breadth across all samples (Figure 4), which corroborates with the results of Mallon et al. (2018). This suggests that O157:H7 invasion facilitated the expansion of this population, which may ultimately lead to a functional change of sediment ecosystem. Further research should include functional genomes to gather deep information on this subject.

Species-time relationship (STR) has frequently been used to analyze microcosm communities in terms of microbial succession (Jiao et al., 2017; Zhang et al., 2020). The STR exponent provides a time-based estimation of the rate of occurrence of new taxa in a community, while a higher exponent indicates more newly introduced taxa (Preston, 1960). Here, the STR exponents of the microcosms (0.002 to 0.025) were lower than the slopes observed in bacterial succession in several natural habitats (Figure 3; Shade et al., 2013). This was attributed to the fact that the relatively closed environment induced by tightly controlled microcosms dramatically reduced the occurrence of new taxa introduced through dispersal. In the controlled experimental system, there was not any recruitment or input from an external species besides the invader (Mallon et al., 2018). Additionally, the STR exponent of the inorganic/organic pollutant-treated microcosms was higher than the microcosms with microbial invader in this study (Jiao et al., 2017). We speculate that the influence of inorganic/organic pollutants was obviously greater than the microbial invader. Also, the abiotic conditions of the microcosms are known to fluctuate due to pollutant degradation (Shade et al., 2013; Jiao et al., 2017), while the abiotic conditions remained constant in this study (data not shown). Despite this, we observed an increase in the STR slope of the total and abundant taxa post-microbial invasion, while the rare taxa remained constant in both $\mathrm{B}$ and $\mathrm{P}$ sediments (Figure 3 ). This indicated that the rare subcommunity have relatively greater stability under the influence of environmental pressure, consistent with other studies (Du et al., 2020). As compared with the abundant taxa, a higher $\alpha$-diversity was observed in the rare taxa which has reported to be taxonomically and functionally diverse (Logares et al., 2014; Du et al., 2020; Zhang et al., 2020). Thus, the hypothesis put forth is that the rare taxa could easily achieve a more stable community after external disturbance. Hence, rare taxa are vital for supporting ecosystem stability against 

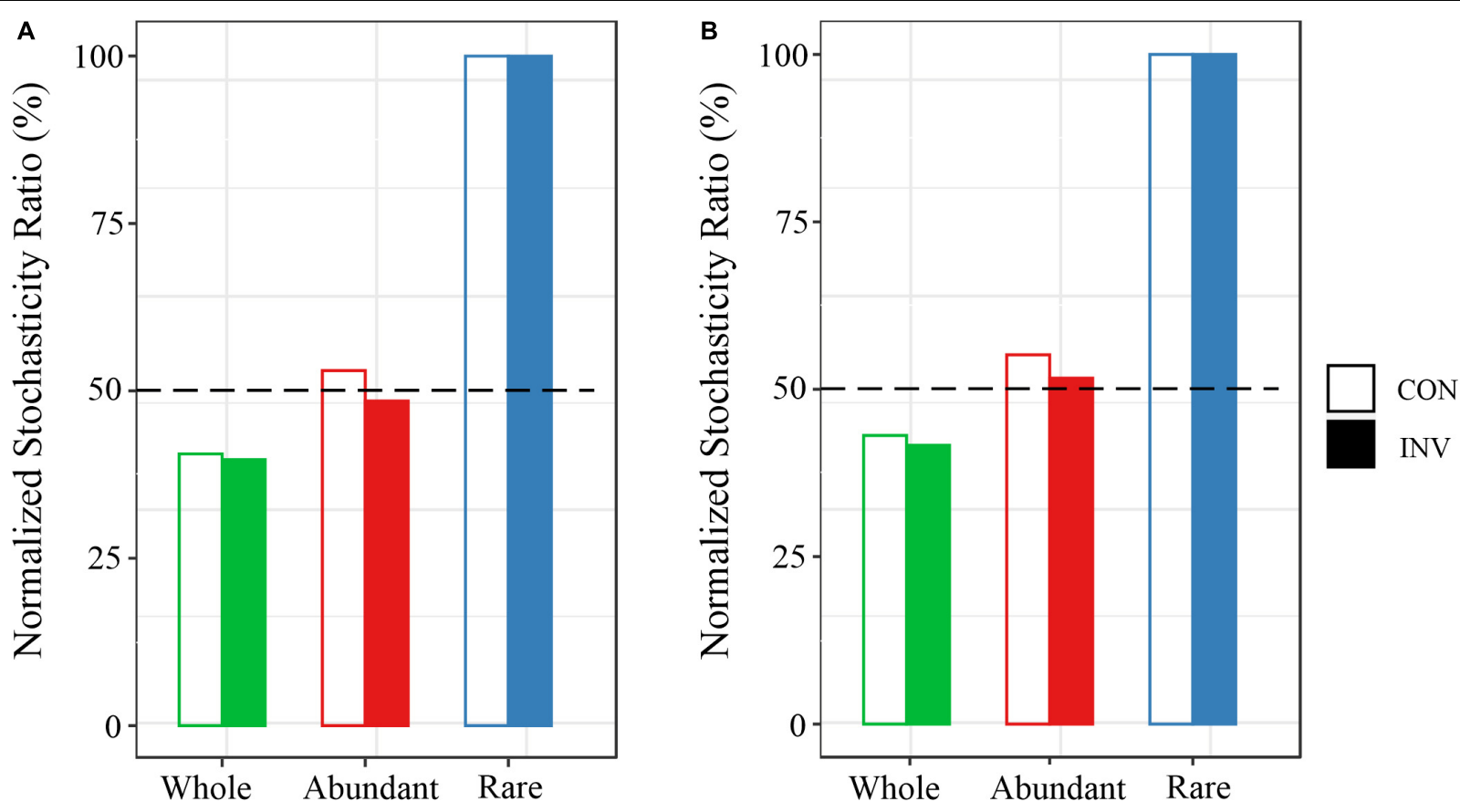

FIGURE 7 | The ecological stochasticity in the assembly of the potentially pathogenic bacterial community evaluated based on the normalized stochasticity ratio (NST) based on the Bray-Curtis indexes. (A) normalized stochasticity ratio of B sediment (B) normalized stochasticity ratio of $\mathrm{P}$ sediment.

microbial invasion and could represent the hidden backbone of the microbial communities (Ziegler et al., 2018).

Network analysis is used to understand the impact of O157:H7 invasion on bacterial co-occurrence pattern. We observed a random arrangement modular framework in the bacterial networks (Table 1, Figures 5, 6, and Supplementary Figure 7), which were suitable to study the co-occurrence modes in the biological systems (Xue et al., 2018). Network topology reflected the variation in the pattern of bacterial interactions between treatments (Table 1). We observed a higher clustering coefficient than that reported for other natural ecosystems (Table 1), including polluted soil (Chao et al., 2016), original marsh sediment (Yao et al., 2019a), and seawater (Zhang et al., 2020), revealing stronger bacterial correlations in this microcosm compared with other ecological networks. This was indicative of niche filtering and cross-feeding relationships in such environment (Ma et al., 2020). Furthermore, the abundant taxa of the CON group exhibited an increased magnitudes of clustering coefficient, density and smaller mean path length than the corresponding INV group (Table 1). The shorter average path length could prompt the perturbations to reach the whole network and contribute to a more efficient system (Zhou et al., 2010; Faust and Raes, 2012; Jin et al., 2020). It is reasonable to infer that the abundant subcommunity of CON group was more complex and efficient since they could cope better with the external changes. Meanwhile, the clustering coefficient and the mean path length remained stable in the rare taxa postinvasion (Table 1), confirming the backbone role of rare taxa in resisting microbial invasion. The results of this study demonstrate that microbial invasion could alter the bacterial co-occurrence pattern, especially the abundant subcommunity, corresponding to a greater variation in bacterial composition of abundant taxa.

In the light of the null model analysis, the results indicated that both deterministic and stochastic assembly regulated assembly of the bacterial community (Supplementary Figures 8, 9). The NST values were used to determine a stochastic $(>50 \%)$ or deterministic $(<50 \%)$ assembly (Ning et al., 2019; Zhou et al., 2020). Here, a stochastic assembly was observed for the rare taxa (NST > 50\%) when compared with the abundant taxa (NST $<50 \%$ ) (Figure 7), which agrees with the results of previous studies (Jiao et al., 2017; Du et al., 2020). We hypothesized that due to their relatively small population size, the demographic stochasticity and dispersal had a significant impact on the rare taxa (Jousset et al., 2017). Moreover, we observed a gradual decrease in the impact of the stochastic processes on the abundant taxa post-microbial invasion (Figure 7), showing the role of homogenizing selection or variable selection increased along with the cumulative effects of O157:H7 invasion. To fully understand the bacterial community assembly mechanisms, future research should consider the sampling scale effects (spatial extent and time scale), more important explanatory deterministic factors (e.g., unmeasured environmental factors and species interactions), and other possible stochastic factors.

\section{CONCLUSION}

Microbial invasions are a threat to ecosystems, and the response of native microbial communities revealed the ecological risk of the microbial invader. Divergent responses of abundant 
and rare sediment taxa to invading E. coli O157:H7 were observed in this study. The rare taxa were found to be more stable to E. coli O157:H7 invasion. Alternatively, there might have been certain changes without an accompanying change in community membership which could not be detected by $16 \mathrm{~S}$ rRNA genes sequencing. Omics technology could be adopted to reveal more detailed changes caused by E. coli O157:H7 invasion. In addition, functional analyses are needed to understand the functional impact of microbial invasion on sediment native microbiota in detail.

\section{DATA AVAILABILITY STATEMENT}

The datasets presented in this study can be found in online repositories. The names of the repository/repositories and accession number(s) can be found below: https://www.ncbi.nlm. nih.gov/, PRJNA669260.

\section{AUTHOR CONTRIBUTIONS}

NZ: writing, formal analysis, and investigation. CL and $\mathrm{XL}$ : investigation and formal analysis. ZY: formal analysis,

\section{REFERENCES}

Amor, D. R., Ratzke, C., and Gore, J. (2020). Transient invaders can induce shifts between alternative stable states of microbial communities. Sci. Adv. 6:8786. doi: $10.1126 /$ sciadv.aay8676

Barberán, A., Bates, S. T., Casamayor, E. O., and Fierer, N. (2012). Using network analysis to explore co-occurrence patterns in soil microbial communities. ISME J. 6, 343-351. doi: 10.1038/ismej.2011.119

Bergmann, G. T., Bates, S. T., Eilers, K. G., Lauber, C. L., Caporaso, J. G., Walters, W. A., et al. (2011). The under-recognized dominance of Verrucomicrobia in soil bacterial communities. Soil Biol. Biochem. 43, 1450-1455. doi: 10.1016/j. soilbio.2011.03.012

Braga, L. P. P., Aymé, S., Kot, W., Breuil, M., and Philippot, L. (2020). Impact of phages on soil bacterial communities and nitrogen availability under different assembly scenarios. Microbiome 8, 828-836. doi: 10.1186/s40168-020-00822-z

Chao, Y. Q., Liu, W. S., Chen, Y. M., Chen, W. H., Zhao, L. H., Ding, Q. B., et al. (2016). Structure, variation, and co-occurrence of soil microbial communities in abandoned sites of a rare earth elements mine. Environ. Sci. Technol. 50, 11481-11490. doi: 10.1021/acs.est.6b02284

Dini-Andreote, F., Stegen, J. C., Van Elsas, J. D., and Salles, J. F. (2015). Disentangling mechanisms that mediate the balance between stochastic and deterministic processes in microbial succession. Proc. Natl. Acad. Sci. U.S.A. 112, 1326-1332. doi: 10.1073/pnas.1414261112

Du, S. C., Dini-Andreote, F., Zhang, N., Liang, C. L., Yao, Z. Y., Zhang, H. J., et al. (2020). Divergent co-occurrence patterns and assembly processes structure the abundant and rare bacterial communities in a salt marsh ecosystem. Appl. Environ. Microb. 86:e0322-20. doi: 10.1128/AEM.00322-20

Faust, K., and Raes, J. (2012). Microbial interactions: from networks to models. Nat. Rev. Microbiol. 10, 538-550. doi: 10.1038/nrmicro2832

Fliessbach, A., Winkler, M., Lutz, M. P., Oberholzer, H. R., and Mader, P. (2009). Soil Amendment with Pseudomonas fluorescens CHA0: lasting effects on soil biological properties in soils low in microbial biomass and activity. Microb. Ecol. 57, 611-623. doi: 10.1007/s00248-009-9489-9

Fukushima, H., and Seki, R. (2004). High numbers of Shiga toxin-producing Escherichia coli found in bovine faeces collected at slaughter in Japan. FEMS Microbiol. Lett. 238, 189-197. doi: 10.1016/j.femsle.2004.07.035

Gao, C. H., Zhang, M., Wu, Y. C., Huang, Q. Y., and Cai, P. (2019). Divergent influence to a pathogen invader by resident bacteria with supervision, writing-review and editing, and investigation. DZ: supervision and writing-review and editing. SD and HZ: formal analysis.

\section{FUNDING}

The work was supported by the National Natural Science Foundation of China (grant numbers 41977348 and 41601517), the Natural Science Foundation of Ningbo (2019A610445), Public Welfare Science and Technology Plan Project of Ningbo (202002N3052), Key Research and Development Program of Zhejiang Province (2020C03082), the Fundamental Research Funds for the Provincial Universities of Zhejiang, and K. C. Wong Magna Fund in Ningbo University.

\section{SUPPLEMENTARY MATERIAL}

The Supplementary Material for this article can be found online at: https://www.frontiersin.org/articles/10.3389/fmicb. 2021.665380/full\#supplementary-material

different social interactions. Microb. Ecol. 77, 76-86. doi: 10.1007/s00248-018$1207-\mathrm{Z}$

Gómez, P. S., Paterson, S., De Meester, L., Liu, X., Lenzi, L., Sharma, M. D., et al. (2016). Local adaptation of a bacterium is as important as its presence in structuring a natural microbial community. Nat. Commun. 7:12453. doi: $10.1038 /$ ncomms 12453

Guo, X., Zhong, H., Li, P., and Zhang, C. J. (2020). Microbial communities responded to tetracyclines and $\mathrm{Cu}(\mathrm{II})$ in constructed wetlands microcosms with Myriophyllum aquaticum. Ecotox. Environ. Safe. 205:111362. doi: 10.1016/j. ecoenv.2020.111362

Hug, L. A., Castelle, C. J., Wrighton, K. C., Thomas, B. C., Sharon, I., Frischkorn, K. R., et al. (2013). Community genomic analyses constrain the distribution of metabolic traits across the Chloroflexi phylum and indicate roles in soil carbon cycling. Microbiome 1:22. doi: 10.1186/2049-2618-1-22

Ishii, S., Ksoll, W. B., Hicks, R. E., and Sadowsky, M. J. (2006). Presence and growth of naturalized Escherichia coli in temperate soils from Lake Superior watersheds. Appl. Environ. Microb. 72, 612-621. doi: 10.1128/AEM.72.1.612-621. 2006

Jia, X., Dini-Andreote, F., and Joana, F. S. (2018). Community assembly processes of the microbial rare biosphere. Trends Microbiol. 26, 738-747. doi: 10.1016/j. tim.2018.02.011

Jiao, S., Chen, W. M., Wang, E. T., Wang, J. M., Liu, Z. S., Li, Y. N., et al. (2016). Microbial succession in response to pollutants in batch-enrichment culture. Sci. Rep. 6:21791. doi: 10.1038/srep21791

Jiao, S., Luo, Y. T., Lu, M. M., Xiao, X., Lin, Y. B., Chen, W. M., et al. (2017). Distinct succession patterns of abundant and rare bacteria in temporal microcosms with pollutants. Environ. Pollut. 225, 497-505. doi: 10.1016/j.envpol.2017. 03.015

Jin, M., Yu, X. B., Yao, Z. Y., Tao, P. R., Li, G., Yu, X. W., et al. (2020). How biofilms affect the uptake and fate of hydrophobic organic compounds (HOCs) in microplastic: insights from an in situ study of Xiangshan Bay, China. Water Res. 184:116118. doi: 10.1016/j.watres.2020.116118

Jousset, A., Bienhold, C., Chatzinotas, A., Gallien, L., Gobet, A., Kurm, V., et al. (2017). Where less may be more: how the rare biosphere pulls ecosystems strings. ISME J. 11, 853-862. doi: 10.1038/ismej.2016.174

Levine, J. M., Adler, P. B., and Yelenik, S. G. (2004). A meta-analysis of biotic resistance to exotic plant invasions. Ecol. Lett. 7, 975-989. doi: 10.1111/j.14610248.2004.00657.x 
Li, S. P., Tan, J. Q., Yang, X., and Jiang, L. (2019). Niche and fitness differences determine invasion success and impact in laboratory bacterial communities. ISME J. 13, 402-412. doi: 10.1038/s41396-018-0283-x

Liang, C. L., Yao, Z. Y., Du, S. C., Hong, M., Wang, K., and Zhang, D. M. (2019). Sediment $\mathrm{pH}$, not the bacterial diversity, determines Escherichia coli O157:H7 survival in estuarine sediments. Environ. Pollut. 252, 1078-1086. doi: 10.1016/j. envpol.2019.06.019

Logares, R., Audic, S., Bass, D., Bittner, L., Boutte, C., Christen, R., et al. (2014). Patterns of rare and abundant marine microbial eukaryotes. Curr. Biol. 24, 813-821. doi: 10.1016/j.cub.2014.02.050

Ma, B., Wang, Y. L., Ye, S. D., Liu, S., Stirling, E., Gilbert, J. A., et al. (2020). Earth microbial co-occurrence network reveals interconnection pattern across microbiomes. Microbiome 8, 1-12. doi: 10.1186/s40168-020-00857-2

Mallon, C. A., Roux, X. L., Van Doorn, G. S., Dini-Andreote, F., Poly, F., and Salles, J. F. (2018). The impact of failure: unsuccessful bacterial invasions steer the soil microbial community away from the invader's niche. ISME J. 12, 728-741. doi: 10.1038/s41396-017-0003-y

Nemergut, D. R., Schmidt, S. K., Fukami, T., O’Neill, S. P., Bilinski, T. M., Stanish, L. F., et al. (2013). Patterns and processes of microbial community assembly. Microbiol. Mol. Biol. R. 77, 342-356. doi: 10.1128/MMBR.00051-12

Ning, D. L., Deng, Y., Tiedje, J. M., and Zhou, J. Z. (2019). A general framework for quantitatively assessing ecological stochasticity. Proc. Natl. Acad. Sci. U.S.A. 116, 16892-16898. doi: 10.1073/pnas.1904623116

Parada, A. E., Needham, D. M., and Fuhrman, J. A. (2016). Every base matters: assessing small subunit rRNA primers for marine microbiomes with mock communities, time series and global field samples. Environ. Microbiol. 18, 1403-1414. doi: 10.1111/1462-2920.13023

Pedros-Alio, C. (2012). The rare bacterial biosphere. Annu. Rev. Mar. Sci. 4, 449-466. doi: 10.1146/annurev-marine-120710-100948

Preston, F. W. (1960). Time and space and the variation of species. Ecology 41, 612-627. doi: 10.2307/1931793

Robin, C., Piou, D., Feau, N., Douzon, G., Schenck, N., and Hansen, E. M. (2011). Root and aerial infections of Chamaecyparis lawsoniana by Phytophthora lateralis: a new threat for European countries. Forest Pathol. 41, 417-424. doi: 10.1111/j.1439-0329.2010.00688.x

Shade, A., Caporaso, J. G., Handelsman, J., Knight, R., and Fierer, N. (2013). A meta-analysis of changes in bacterial and archaeal communities with time. ISME J. 7, 1493-1506. doi: 10.1038/ismej.2013.54

Shu, D. T., Yue, H., He, Y. L., and Wei, G. H. (2018). Divergent assemblage patterns of abundant and rare microbial sub-communities in response to inorganic carbon stresses in a simultaneous anammox and denitrification (SAD) system. Bioresource Technol. 257, 249-259. doi: 10.1016/j.biortech.2018.02.111

Thakur, M. P., Der, P. W. H., Cobben, M. M., Kleunen, M. V., and Geisen, S. (2019). Microbial invasions in terrestrial ecosystems. Nat. Rev. Microbiol. 17, 621-631. doi: 10.1038/s41579-019-0236-z

Van Elsas, J. D., Semenov, A. V., Costa, R., and Trevors, J. T. (2011). Survival of Escherichia coli in the environment: fundamental and public health aspects. ISME J. 5, 173-183. doi: 10.1038/ismej.2010.80

Wei, Z., Jie, H., Gu, Y., Yin, S. X., and Xu, Y. C. (2018). Ralstonia solanacearum pathogen disrupts bacterial rhizosphere microbiome during an invasion. Soil. Biol. Biochem. 118, 8-17. doi: 10.1016/j.soilbio.2017.11.012

Wei, Z., Yang, T. J., Friman, V., Xu, Y. C., Shen, Q. R., and Jousset, A. (2015). Trophic network architecture of root-associated bacterial communities determines pathogen invasion and plant health. Nat. Commun. 6:8413. doi: $10.1038 /$ ncomms 9413
Wu, J. Y., Gu, L., Hua, Z. L., Li, X. Q., Lu, Y., and Chu, K. (2021). Effects of Escherichia coli pollution on decomposition of aquatic plants: variation due to microbial community composition and the release and cycling of nutrients. J. Hazard. Mater. 401:123252. doi: 10.1016/j.jhazmat.2020.123252

Xing, J. J., Sun, S. S., Wang, H. Z., Brookes, P. C., and Xu, J. M. (2020). Response of soil native microbial community to Escherichia coli O157:H7 invasion. Environ. Pollut. 261:114225. doi: 10.1016/j.envpol.2020.114225

Xue, Y. Y., Chen, H. H., Yang, J. R., Liu, M., Huang, B. Q., and Yang, J. (2018). Distinct patterns and processes of abundant and rare eukaryotic plankton communities following a reservoir cyanobacterial bloom. ISME J. 12, 22632277. doi: 10.1038/s41396-018-0159-0

Yao, Z. Y., Du, S. C., Liang, C. L., Zhao, Y. J., Andreote, F. D., Wang, K., et al. (2019a). Bacterial community assembly in a typical estuarine marsh with multiple environmental gradients. Appl. Environ. Microb. 85, e2602-e2618. doi: 10.1128/AEM.02602-18

Yao, Z. Y., Wang, H. Z., Wu, L. S., Wu, J. J., Brookes, P. C., and Xu, J. M. (2014). Interaction between the microbial community and invading Escherichia coli O157:H7 in soils from vegetable fields. Appl. Environ. Microb. 80, 70-76. doi: 10.1128/AEM.03046-13

Yao, Z. Y., Wei, G., Wang, H. Z., Wu, L. S., Wu, J. J., and Xu, J. M. (2013). Survival of Escherichia coli O157:H7 in soils from vegetable fields with different cultivation patterns. Appl. Environ. Microb. 79, 1755-1756. doi: 10.1128/AEM.03 605-12

Yao, Z. Y., Zhang, H. J., Liang, C. L., Wang, Y. Z., and Wu, Y. P. (2019b). Effects of cultivating years on survival of culturable Escherichia coli O157:H7 in Greenhouse soils. J. Food Protect. 82, 226-232. doi: 10.4315/0362-028X.JFP18-287

Zhang, H. J., Hou, F. R., Xie, W. J., Wang, K., Zhou, X. Y., Zhang, D. M., et al. (2020). Interaction and assembly processes of abundant and rare microbial communities during a diatom bloom process. Environ. Microbiol. 22, 17071719. doi: 10.1111/1462-2920.14820

Zhou, J. Z., and Ning, D. L. (2017). Stochastic community assembly: does it matter in Microb. Ecol. Microbiol. Mol. Biol. R. 81:e00002-e17. doi: 10.1128/mmbr. 00002-17

Zhou, J. Z., Deng, Y., Luo, F., He, Z. L., Tu, Q. C., and Zhi, X. Y. (2010). Functional molecular ecological networks. mBio 1:e00169-10. doi: 10.1128/mBio.00 169-10

Zhou, L., Li, L., Chen, W. Y., Sun, J. J., Hou, S. W., Kuang, T. X., et al. (2020). Stochastic determination of the spatial variation of potentially pathogenic bacteria communities in a large subtropical river. Environ. Pollut. 264:114683. doi: 10.1016/j.envpol.2020.114683

Ziegler, M., Eguiluz, V. M., Duarte, C. M., and Voolstra, C. R. (2018). Rare symbionts may contribute to the resilience of coral-algal assemblages. ISME J. 12, 161-172. doi: 10.1038/ismej.2017.151

Conflict of Interest: The authors declare that the research was conducted in the absence of any commercial or financial relationships that could be construed as a potential conflict of interest.

Copyright (C) 2021 Zhang, Liang, Liu, Yao, Zhu, Du and Zhang. This is an open-access article distributed under the terms of the Creative Commons Attribution License (CC BY). The use, distribution or reproduction in other forums is permitted, provided the original author(s) and the copyright owner(s) are credited and that the original publication in this journal is cited, in accordance with accepted academic practice. No use, distribution or reproduction is permitted which does not comply with these terms. 